\title{
Inventario de sitios arqueológicos en el municipio de Rosita
}

\section{Kevin E. González Hodgson Edwin Taylor Rigby}

Con la documentación y el registro de sitios arqueológicos que aquí presentamos esperamos contribuir a que el municipio de Rosita, en la Región Autónoma Atlántico Norte (RAAN), tenga un inventario inicial de sitios de interés arqueológico. Para esto fue necesario implementar, además de la recolección de materiales antiguos en superficie, una metodología propia y consistente de prospección arqueológica ${ }^{l}$.

El estudio se desarrolló concretamente en el casco urbano de Rosita y en las localidades de La Florida, El Zopilote, Minnesota y Susun Central/Arriba. La prospección se dio durante los meses de abril y mayo del 2012, período en que se pudo identificar un total de 17 sitios arqueológicos. Los sitios en mención se caracterizan por presentar una serie de evidencias materiales-culturales, indicadoras del desarrollo de asentamientos indígenas ${ }^{2}$ en la región. Las evidencias materiales identificadas corresponden a estructuras monticulares, petroglifos, estatuarias, artefactos líticos y tiestos cerámicos del tipo monocromo dispuestos en superficie. Estos hallazgos fueron motivos suficientes para desarrollar este estudio.

\section{ANTECEDENTES DE ESTUDIO}

Muchas son las evidencias reportadas de sitios arqueológicos tanto en el Caribe Norte como en el Caribe Sur de Centro América. En el caso del Caribe nicaragüense, muchos de estos lugares no han sido documentados, excavados ni reportados y, sin duda, la cantidad de sitios sin conocer es grande (Balladares y Lechado, 2007, 38). Probablemente, los numerosos recursos naturales propiciaron el desarrollo de asentamientos humanos de manera constante en la región centroamericana. Algunas recopilaciones y estudios realizados con anterioridad por Gassiot, (1993:3-4), y referido por Balladares y Lechado (2007:38), evidencian este antiguo poblamiento en toda la región del Caribe centroamericano.

Haciendo referencia a la Región Autónoma Atlántico Norte en lo que respecta a estudios estrictamente arqueológicos, no es sino hasta el 2006 - 2007 y 2008 que se ejecutó la primera investigación -específica, para ubicar sitios arqueológicos en esta región; dicho estudio será abordado más adelante. Previo a este estudio, lo que se conocía era lo planteado por investigadores como Edward Conzemius, Gotz Von Houwald, Guido Grossman, W. Haberland, Luis Hurtado de Mendoza y Walter Lehmann, quienes reportaban evidencias arqueológicas encontradas y observadas durante sus investigaciones; dichas evidencias correspondían a tiestos cerámicos, piedras de moler y petroglifos.

Entre los mencionados anteriormente, Götz Von Houwald y W. Haberland son los únicos que realizaron lo que podría ser catalogado como una investigación arqueológica. Sin embargo, su investigación titulada Cerro Mokó, ein heiliger berg der Sumo-indianer? Aún no se ha traducido al español.

Sumado a toda la evidencia expuesta con anterioridad cabe indicar que también existen publicaciones inéditas, referencias orales y fotografías, sobre evidencias arqueológicas encontradas en distintos territorios de la región. Algunas de las más significativas fueron obtenidas por el arqueólogo nicaragüense Bayardo Gámez y el antropólogo estadounidense Jaime Feltz en1997. La importancia de la investigación radica en que existe un diario de campo que contiene todos los lugares en que se reporta la existencia de material arqueológico.

$1 \quad$ Método que consiste en supervisar la superficie del terreno determinado, con el fin de encontrar evidencias culturales que permitan saber si en dicha área se desarrollaron o no antiguos asentamientos humanos (Renfrew y Bahn, 1993:67).

2 Lugares donde se encuentran conjuntos de evidencias materiales que reflejan actividades de grupos humanos sedentarios, a la vez que reflejan el nivel de desarrollo y organización de éstos (Lange, 1993:4). 


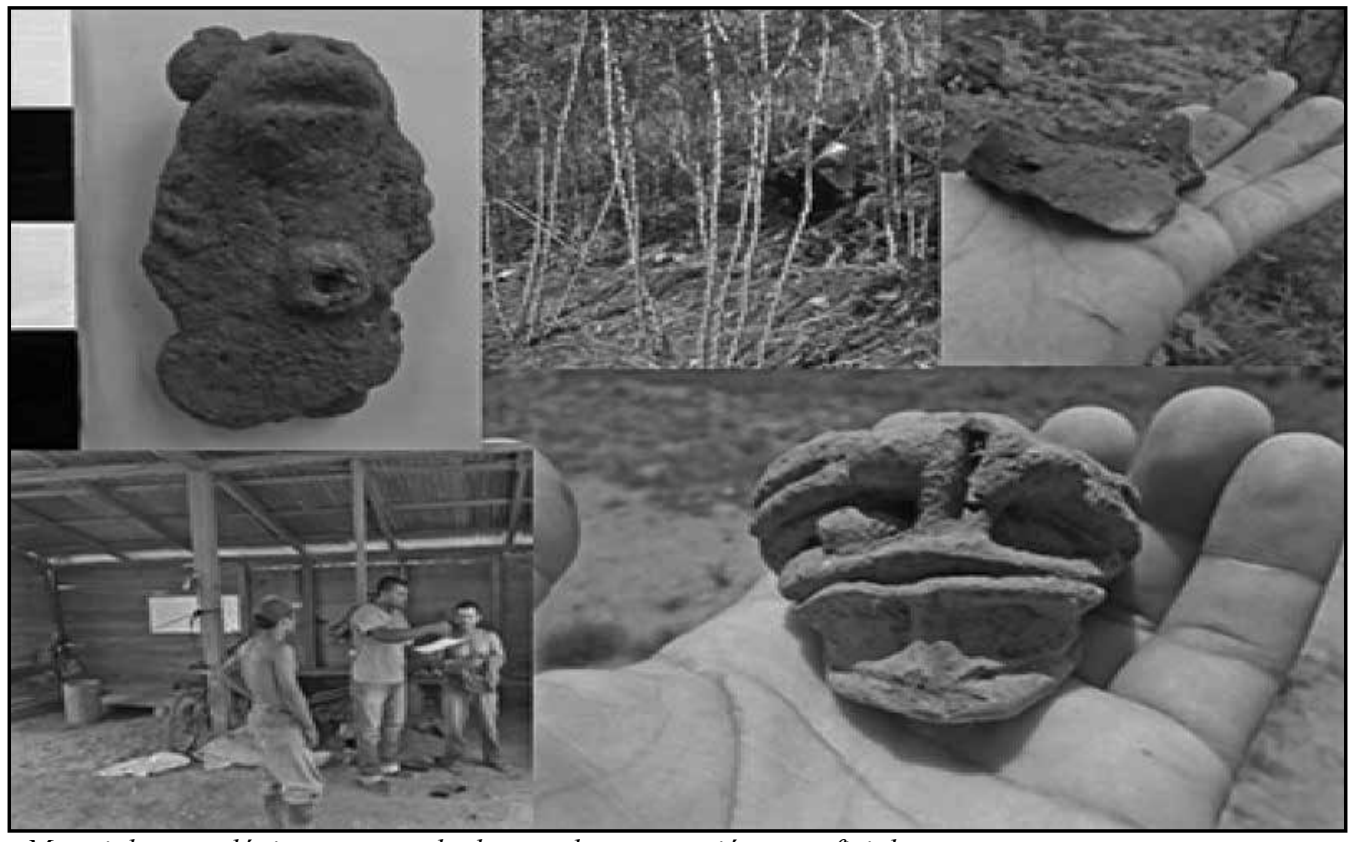

Material arqueológico encontrado durante la prospección superficial.

Los mencionados estudios arqueológicos de los años 2006, 2007 y 2008 incluyeron, en el 2006, un reconocimiento de la comunidad La Florida, en el municipio de Rosita, identificándose evidencia arqueológica, lo que posteriormente se tradujo en la documentación y registro de varios sitios arqueológicos en esta comunidad. Posteriormente se amplía el horizonte de estudio con la investigación llevada a cabo por los arqueólogos Kevin González Hodgson y Edwin Taylor Rigby, bajo el titulo de Arqueología en la RAAN: Una aproximación al pasado, donde se logra documentar, mediante una prospección en los municipios de Waspam, Bonanza, Prinzapolka y Rosita, un total de 20 sitios arqueológicos ubicados en este municipio en su mayoría.

Pero también es válido tomar en cuenta el reciente reconocimiento de material arqueológico en La Florida en el 2011, desarrollado por el arqueólogo Ramiro García Vázquez, cuyo informe fue de gran utilidad para nuestra investigación.

Todo lo anteriormente expuesto refleja la riqueza arqueológica que posee esta parte del territorio nicaragüense y, en este caso, el territorio de Rosita. Sin embargo, solo con investigaciones arqueológicas sistemáticas continuas se podrá elaborar un plan de acción en pro del resguardo, conservación y protección del patrimonio arqueológico.

\section{RESULTADOS}

Aunque la propuesta estaba destinada a investigar cuatro comunidades (El Zopilote, La Florida, Minnesota, Susun Central/Arriba) y propiamente el casco urbano de Rosita, sin embargo se prospectó otras regiones próximas a las comunidades dispuestas (entre ellas Arenaloso, Cerro La Unión y San Francisco de Banacruz), debido a que los mismos pobladores hicieron referencias a evidencias arqueológicas en dichos territorios. En el caso de Susun Arriba se visitó esta comunidad, sin embargo, las condiciones climáticas no permitieron determinar sitios de interés arqueológicos en dicha zona.

La prospección arqueológica, como método empleado para la elaboración del inventario de sitios, permitió registrar y documentar un total de 17 sitios arqueológicos en el municipio. La muestra de cerámica estuvo conformada por un total de 240 elementos recolectados en los municipios trabajados, de los cuales, en relación al tipo de evidencia registrada y tomando en cuenta aspectos del medio físico, fueron clasificados de la siguiente manera:

\section{Ubicación de los sitios}

La mayoría de los sitios (12 de 17) documentados en esta investigación estaban situados en zonas altas. Existe información de otros sitios documentados con

3 Uno de los sitios documentados con este tipo de características es el cerro Moko, localizado en Santa Fe, rio Coco, municipio de Waspam. 
características similares en la región ${ }^{3}$, que viene a corroborar patrones similares en cuanto al emplazamiento empleado en estos sitios.

\section{Tipos de sitios}

Luego de haber caracterizado los sitios inventariados de acuerdo a su distribución espacial, se estableció definirlos acorde a las particularidades en relación a la evidencia que presentaban cada uno de ellos. De esta forma se pudo identificar 12 sitios con presencia de material en superficie; un sitio con material en superficie, pero con el agregado de estructuras monticulares; tres sitios con petroglifos, y un solo sitio con estatuilla. La tipificación de los sitios, sobre todo de los dos primeros, quizás no difieran mucho entre sí, puesto que el primero pudo haber contemplado las mismas características del segundo, lo que sugiere es que pudieron haberse desarrollado actividades concretas en la zona, como son los asentamientos, y que en relación a los petroglifos y estatuilla documentados formaban parte de las manifestaciones artísticas desarrolladas en aquel entonces.

\section{Recolección de las muestras y resultados de su procesamiento}

En los 17 sitios documentados y registrados durante la jornada de campo fueron tomadas muestras diagnósticas del material arqueológico, que en su mayoría correspondían a tiestos cerámicos, además de material lítico en superficie. En base a sus características, dichas muestras fueron lavadas, clasificadas y analizadas en laboratorio, obteniendo los siguientes resultados de ese procedimiento:
La forma de la cerámica definirá su funcionalidad/uso, porque su fabricación obedece a un fin preciso. Para esto se toman en cuenta elementos como el ancho de la boca de las vasijas, capacidad de contenido, grosor, entre otros aspectos que se determinan por medio del análisis en laboratorio.

De los atributos de la cerámica y el análisis morfométrico realizado a las mismas se pudo interpretar, de forma general, que las muestras representan, mayoritariamente, cerámica del tipo utilitaria, es decir, utilizada probablemente para actividades domésticas como almacenamiento y/o cocción de alimentos.

Del total de la muestra cerámica recolectada por cada comunidad y según el tratamiento de su superficie, el comportamiento de ésta se caracterizó de la siguiente manera:

La evidencia cerámica apunta a demostrar que la zona no estuvo deshabitada remotamente sino todo lo contrario, tal y como lo refleja la variada evidencia documentada. El barrio 28 de Mayo, que forma parte del casco urbano de Rosita, se documentó como el sitio de mayor frecuencia en relación a materiales arqueológicos en superficie.

Importante también es considerar el inventario que se hizo a la colección particular de dos pobladores del municipio de Rosita, uno situado en el casco urbano y otro ubicado en la comunidad de El Zopilote.

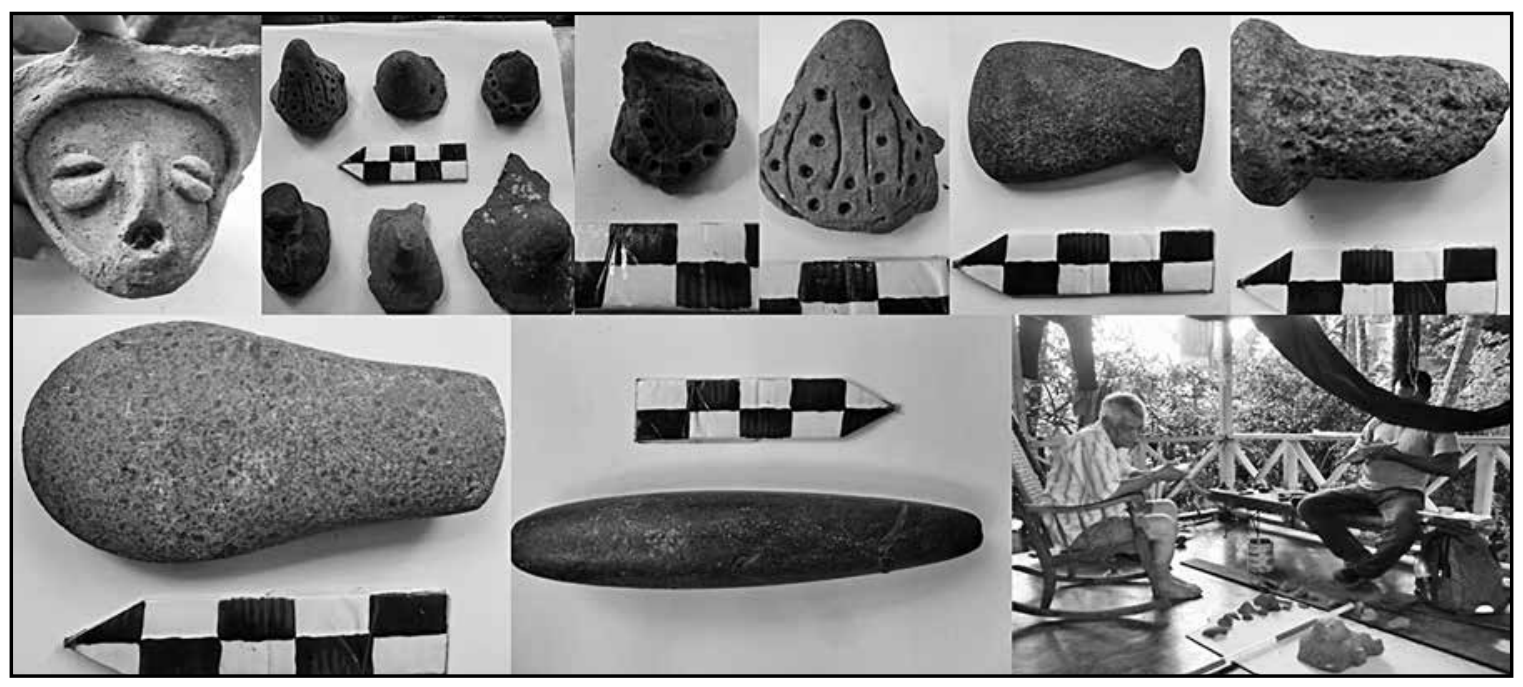

Piezas arqueológicas encontradas por algunos coleccionistas locales como el profesor Adán Silva. 
Cabe destacar que estas referencias son de suma importancia para el inventario de sitios ya que si bien es cierto que no se puede tener claro el contexto arqueológico del cual provienen las piezas, al menos permiten conocer, de alguna manera, las técnicas de decoración y manufactura en el proceso de su elaboración, así como también demuestra la variada y dispersa evidencia que se encuentra en el Municipio.

\section{Técnicas de decoración y relaciones con otras cerámicas}

Existían diferentes técnicas para decorar las vasijas. En algunas ocasiones se utilizaba una sola; en otras se combinaban dos o más, por ejemplo, la incisión ${ }^{4}$ con pintura o con punzonado, o bien, el alisado con engobe, por lo que la utilización de técnicas tan diversas hace que, por lo general, los diseños sean complicados. La mayoría de los tiestos cerámicos analizados están alisados ${ }^{5}$ y presentan decoración por impresiones. Estas características reflejan en parte el tipo de técnicas de decoración empleado en las vasijas cerámicas. De este tipo de técnica se tiene conocimiento en muchas de las cerámicas de los sitios de El Cascal, Flor de Pino y Caroline localizados en el municipio de Kukra Hill, en la Región Autónoma Atlántico Sur.
Al igual que la cerámica, los materiales líticos recolectados también fueron analizados. Este material corresponde a cinco lascas, dos fragmentos micropulidos y un bifaz. Al menos, en la muestra analizada no hay tendencia de una producción dirigida a la búsqueda de piezas con filos rugosos y se determinó la percusión como técnica empleando básicamente un percutor duro.

En base al análisis realizado en las muestras de cerámicas, y debido a la ausencia de documentación bibliográfica relacionada con la zona, establecimos asociaciones o análisis comparativos entre las muestras arqueológicas y sitios documentados en la región del valle de Comayagua, en Honduras. Técnicas de decoración (incisiones, impresiones y aplicaciones) utilizadas por los indígenas de Comayagua se lograron identificar en las muestras, lo que sugiere, hipotéticamente, la aplicación de patrones de decoraciones similares. Sin embargo, esto es solo un eventual planteamiento y sin duda es necesario esperar en un futuro los resultados de un estudio más exhaustivo de los materiales, que permitan una evaluación global de los sitios.

\section{Características cerámicas}
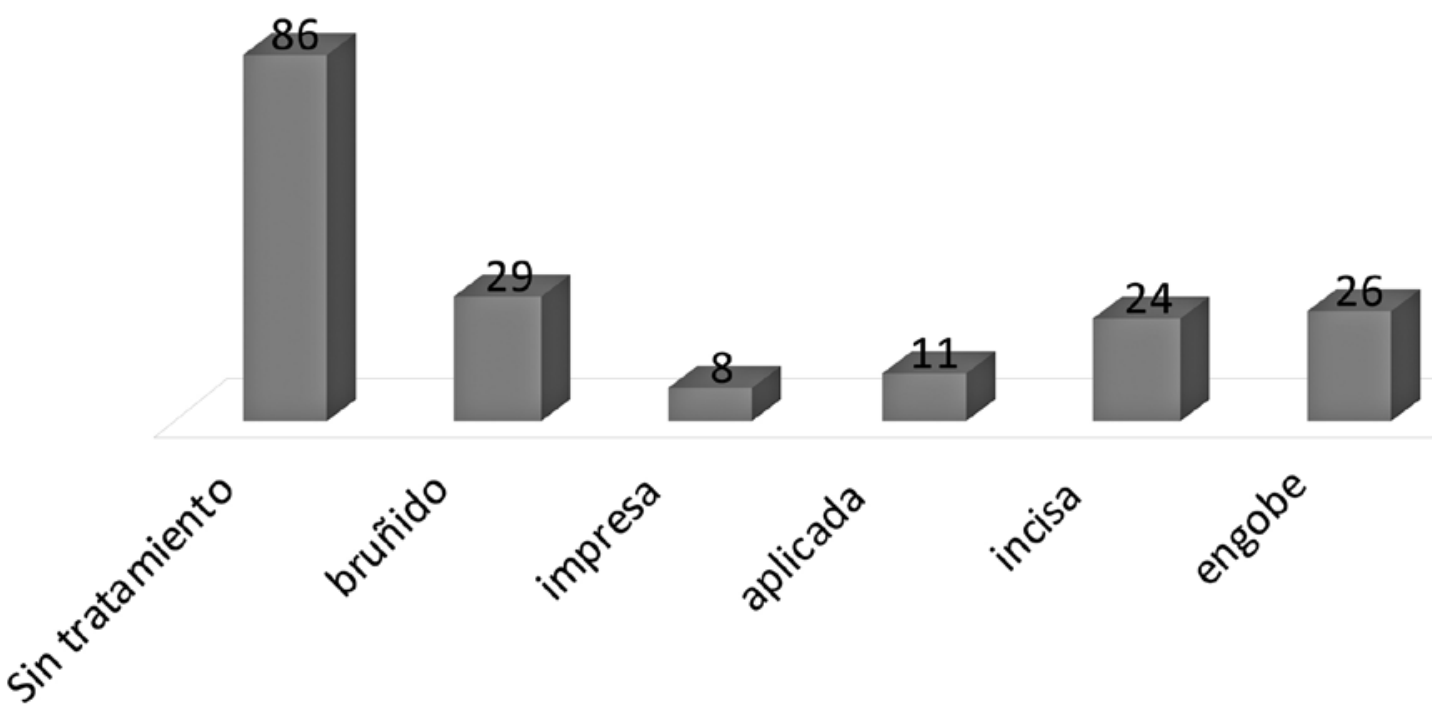


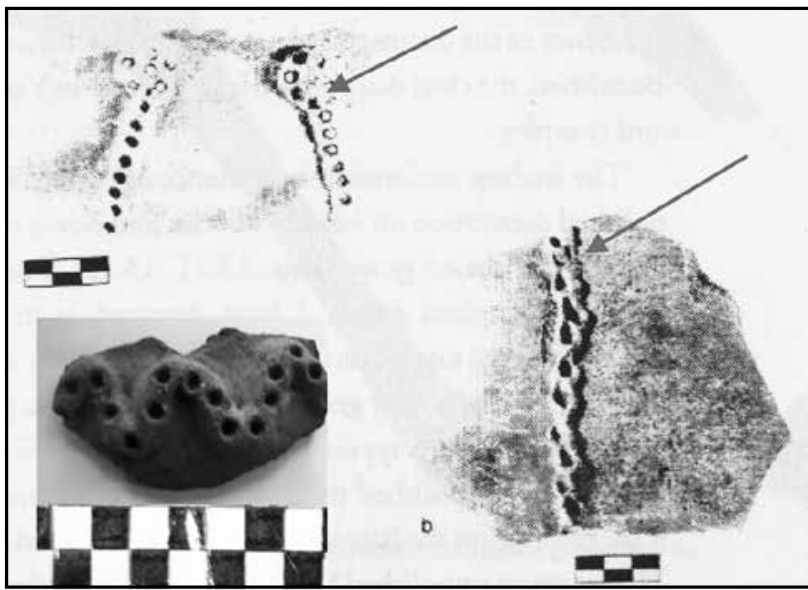

Cerámica de Honduras "Yarumela complex", y cerámica del sitio (ED1), La Florida (inserto). Tomado de Pottery of Prehistoric Honduras: Regional classification and analysis.

Así mismo se pudo determinar similitudes entre las técnicas de decoración -en cuanto a impresiones- encontradas en soportes cerámicos procedentes de Laguna de Perlas (RAAS) y los descubiertos en la comunidad La Florida, en el municipio de Rosita.

El mapa que a continuación se observa refleja la distribución espacial que tienen los sitios arqueológicos en el municipio de Rosita, nótese el extenso territorio que aún falta prospectar.

La evidencia analizada en laboratorio junto al registro de montículos, petroglifos y estatuarias indican el amplio poblamiento antiguo que hubo en el pasado en la región del Caribe y particularmente en el municipio de Rosita.

\section{Relación de sitios arqueológicos}

A continuación hacemos referencia a los datos obtenidos de las prospecciones arqueológicas. En la mayoría de los casos son lugares arqueológicos inéditos en los cuales no se ha realizado ningún tipo de estudio. Algunos de éstos son conocidos únicamente por los pobladores de la zona. También recogemos ciertos sitios ya conocidos en la bibliografía arqueológica consultada y otros cercanos al área de estudio.

\section{La Florida \# 1}

Material cerámico lítico en superficie. (N-RAAN-Rta-LF6 ${ }^{1}$ ).

Presenta material cerámico frecuente como: cuerpos, bordes y soportes, junto a material lítico escaso y en disposición dispersa. Entre el material lítico encontrado se puede mencionar un fragmento (sílex). Se encuentra en la cima de una montaña con pendiente suave. Algunas de las muestras han sido recuperados en la superficie de las laderas y en la propia cima. Sobre él se encontraron restos de una casa cuya ocupación alteró al sitio en alguna medida.

\section{La Florida \# 2}

Estructura monticular (N-RAAN-Rta-LF7).

El sitio presenta tres estructuras monticulares ${ }^{2}$ compuestas de piedras y tierra, las cuales se encuentran cubiertas por la maleza, lo que impidió que se detallaran mejor; el diámetro entre ellas varía de una a otra, destacando,

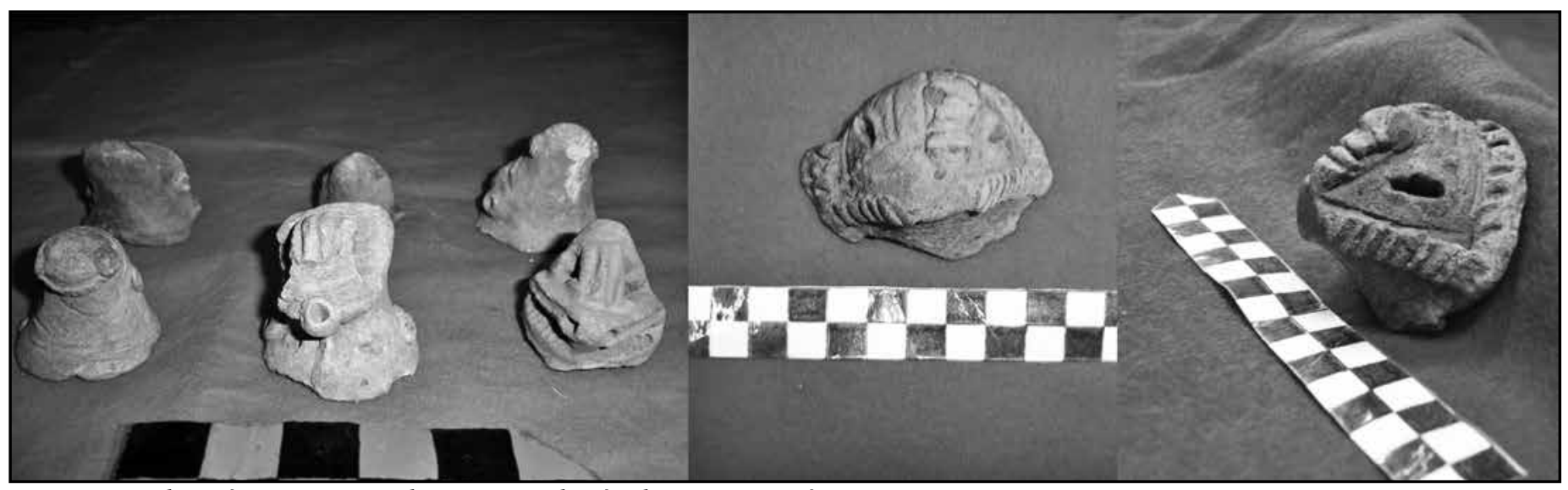

Fragmentos de cerámica correspondientes a pies de trípodes en su mayoría.

$1 \quad$ Los códigos que aparecen en cada sitio responden a la nomenclatura/códigos asignados para el inventario de sitios arqueológicos en la RAAN, facilitados por la Dirección de Patrimonio Cultural a través del Departamento de Arqueología.

2 Estructuras monticulares o monticulos: Acumulaciones intencionadas de piedras y/o tierra, que facilitaban el desarrollo de actividades concretas (habitación, centros ceremoniales, etc.) en algunos grupos humanos. 
una con dimensiones de más de diez metros en su radio de alcance. Se identificó presencia de diversos tipos de materiales en su superficie, entre otros: tiestos cerámicos, con una frecuencia dominante en el terreno (bordes, cuerpos y soportes o "chichas"), en cantidades considerables y en disposición agrupada. Se encontró, además, cerámica decorada e incisa (frecuentes), aplicadas (escasas) y esculpidas (dominantes). Con respecto a la lítica, no se logró encontrar ninguna muestra en el terreno, ya que la vegetación y la gran cantidad de desechos orgánicos (hojas, ramas, maleza), no permitieron valorar el material a simple vista.

\section{La Florida \# 3}

Material cerámico en superficie/ estatuaria.

(N-RAAN-Rta-LF8)

Se definió como sitio en función de la presencia de material cerámico fragmentado, dominante y disperso, con predominio de cerámica cuerpo $\mathrm{y}$, en menor cantidad, soportes y bordes presentando incisiones; en relación al tratamiento de la superficie no presentan decoración, ya que la mayoría del material es monocromo. En el sitio también se halló una piedra que presenta figuras en sus paredes, cuyo tamaño aproximado es de medio metro, donde hay que señalar que la parte interna del grabado posiblemente sea reciente, aunque los bordes exteriores del mismo sean de origen antiguo, por lo que no se descarta la posibilidad que la misma sea una estatuaria/ escultura, producto de la técnica de talla antigua.

\section{La Florida \# 4}

Material cerámico/lítico en superficie.

(N-RAAN-Rta-LF9)

El terreno no es recomendado para la recolección de muestras, sin embargo se punteó el sitio con GPS. El material cerámico era escaso, con presencia de fragmentos de cuerpos con engobe; en cuanto a la lítica se pudo determinar dos lascas (talla).

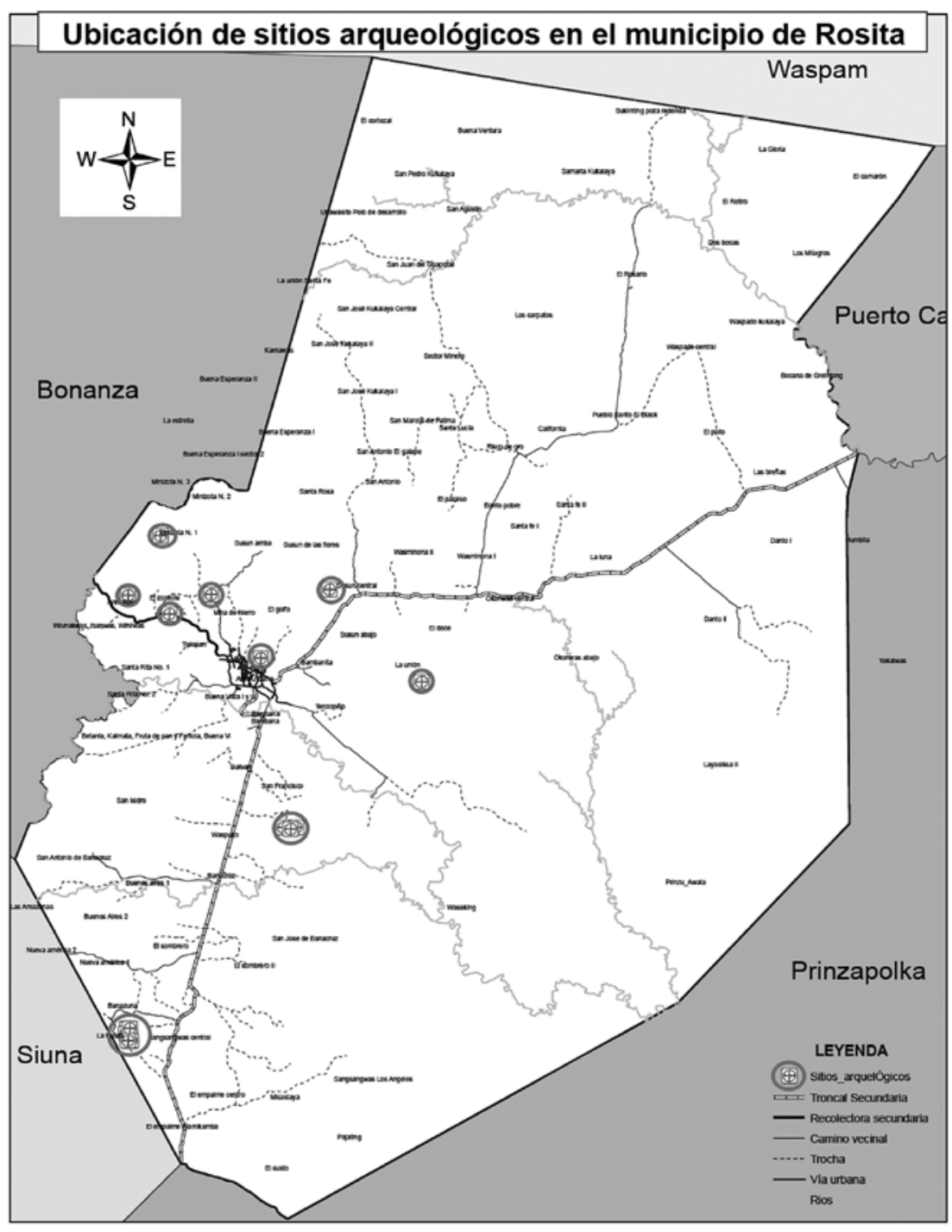

\section{La Florida \# 5}

Petroglifo (N-RAAN-Rta-LF10)

Presencia de una roca con petroglifo o grabado en piedra, la cual se encuentra en una zona alta de ladera en un cerro que mide 209 m.s.n.m. Se encontró material cerámico en disposición dispersa y con mucha frecuencia en pequeñas partes llanas del cerro, lugar donde se observó la cerámica alterada debido a las actividades de roza y quema. A pesar de que fue documentado el petroglifo, no fue posible definir los motivos grabados, debido al desgaste de la roca. No se descarta la posibilidad de que existan otros que no fueron observables debido a lo denso de la vegetación que cubría a las rocas, sobre todo en el extremo noreste del cerro. 


\section{La Florida \# 6:}

Estatuaria (N-RAAN-Rta-LF11)

El sitio está compuesto por la presencia de una única piedra trabajada de granito cuyas dimensiones oscilan entre 45 centímetros de largo por 20 de ancho. Posiblemente se trate de una estatuaria tallada en piedra, ya que presenta algunas señales de talla, rasgos que no sugieren un patrón definido.

\section{La Florida \# 7}

Petroglifo (N-RAAN-Rta-LF12)

Fueron localizados tres petroglifos ${ }^{3}$ con motivos antropomorfos. El petroglifo documentado está cercano a la casa de habitación de don Juan Díaz, a unos treinta y cinco metros. Es la única muestra que se pudo extraer del sitio ya que las condiciones climáticas no lo permitieron en esa ocasión.

\section{Casco Urbano \# 8}

Material cerámico en superficie (N-RAAN-Rta-Rta13)

Las evidencias encontradas en el sitio fueron abundantes, la mayoría se trataba de material cerámico como: soportes, cuerpos, asas, bordes, entre otros. También se pudo encontrar en la cima del mismo cerro dos posibles montículos bien definidos, sin embargo, debido a la actividad agrícola en el área fue imposible la identificación de otros.

\section{Susun Central \# 9}

Material cerámico en superfície (N-RAAN-Rta-Sunc14)

En cuanto a la evidencia arqueológica encontrada se tiene únicamente tiestos cerámicos localizados en una parte plana de la ladera alta del cerro, en la cual existen, además, posibles montículos similares a los encontrados en el cerro cercano a Rosita (Rta-Rta13). Esto no pudo ser constatado, por lo espeso de la maleza la cual no permitió visualizar dichas formaciones. Se habla de montículos por la razón de encontrar una alineación de piedras que únicamente en futuras investigaciones podrían resolver las expectativas que tenemos ahora en cuanto al sitio.

\section{San Francisco de Banacruz \# 10}

Material cerámico en superficie (N-RAAN-Rta-Sf15)

Existe presencia de material arqueológico en disposición dispersa, sobre todo, muestras diagnósticas como bordes, soportes con características incisas y aplicadas, además de cuerpos sin ninguna técnica de decoración.

\section{San Francisco de Banacruz \# 1}

Petroglifo (N-RAAN-Rta-Sf16)

Presencia de dos rocas de tipo basalto cuyo diámetro es de $1.50 \mathrm{~m}$. Se observa una figura con características geométricas en espiral. Mide $30 \mathrm{~cm}$ de largo por $15 \mathrm{~cm}$ de ancho. Usaron técnica propia de percusión para grabar la superficie de la roca. Ésta mide entre 80 y $90 \mathrm{~cm}$. Se observa en ella grabados o figuras abstractas. El surco de los petroglifos oscila entre 1 y $1.5 \mathrm{~cm}$. En el sitio, según los vecinos, en época de siembra se encuentra cerámica del tipo que hemos reportado en el sitio anterior a éste, cosa que no pudimos corroborar debido a la densa maleza que cubría el lugar.

\section{San Francisco de Banacruz \# 12}

Material cerámico en superficie (N-RAAN-Rta-Sf17)

Se logró identificar tiestos cerámicos en la trocha que conduce hacia la vivienda del propietario, entre ellos destaca uno uno con incisiones. Según el propietario del sitio es común que, durante las actividades agrícolas, se encuentren tiestos cerámicos del tipo soporte, llamado por los pobladores "chichitas de barro".

\section{Minnesota \# 13}

Material cerámico en superficie (N-RAAN-Rta-Mina18)

Se encontró, entre las raíces de un árbol caído, poco material cerámico del tipo bordes y soportes, sin ninguna técnica de decoración. Prospectando el área detenidamente se constató la presencia de más evidencia cerámica, como bordes y soportes, en el patio de una vivienda próxima. También se observó, cerca a la vivienda, lo que posiblemente sea un estructura monticular y un fragmento de piedra de moler (metate) de aproximadamente unos 25 $\mathrm{cm}$ de ancho por $20 \mathrm{~cm}$ de largo.

\section{Mina de Hierro, Minnesota\# 14}

Material cerámico en superficie (N-RAAN-Rta-Mina19)

En el patio de la casa de una persona identificada como doña Yelba se pudo observar tiestos cerámicos en poca densidad tales como fragmentos de cuerpos y bordes, algunos con tratamiento en la superficie, como aplicaciones e incisiones.

Petroglifos: Grabados en piedras de gran tamaño que elaboraron las sociedades pasadas y que de alguna u otra manera expresaban su arte como grupo. 


\section{Cerro La Unión \# 15}

Material cerámico en superficie (N-RAAN-Rta-Uno 20)

Presencia de materiales tales como soportes y cuerpos que presentan técnicas decorativas de incisiones. El material en superficie es abundante y la evidencia aparece como resultado de actividades de derrumbe/deslave de un sector del cerro con el paso del huracán Ida en 2009. El tipo de suelo existente se caracteriza por ser arcilloso-arenoso, poco fértil para actividades de siembra.

\section{El Zopilote \# 16}

Material cerámico en superficie (N-RAAN-Rta- Zpl 20)

Presencia de abundante material cerámico en superficie, dispuesto en forma dispersa a lo largo de aproximadamente cinco manzanas, generalmente tiestos del tipo bordes, soportes y muchos cuerpos.

\section{Arenaloso \# 17}

Material cerámico en superficie (N-RAAN-Rta- Arena 21)

Presencia de abundante material cerámico en superficie sobre todo de tiestos cerámicos del tipo bordes, y soportes y cuerpos con técnicas de decoración incisas.

\section{Estado de conservación de los sitios y materiales arqueológicos}

La mayoría de los sitios documentados en este inventario están siendo impactados por la actividad agrícola y ganadera muy común en la zona. Importante también es señalar el perjuicio que causan en los materiales arqueológicos los agentes atmosféricos y climáticos (como el desgaste en la superficie de la cerámica) que hacen que rasgos como la decoración, aplicación, pintura y otros elementos de interés, apenas se perciban. Por otra parte, los suelos en la región se caracterizan por ser ácidos, de manera que también jugaron un papel determinante en el deterioro de los materiales, ya que éstos tienden a oxidarse con el tiempo, o bien los tiestos se disponen en forma porosa.

\section{CONCLUSIONES}

Respecto a la recién campaña de investigación ejecutada en el municipio de Rosita, ésta ha dejado mas enigmas que antes de iniciarla, puesto que la región no ha sido estudiada de manera sistemática en lo relacionado a estudios arqueológicos.

Empleando en el inventario el método de prospección arqueológica en siete comunidades del municipio de
Rosita se obtuvo la documentación y registro de 17 sitios arqueológicos, distribuidos éstos entre estructuras monticulares (2), material en superficie (12), petroglifos (3) y estatuillas (1).

Fue de suma importancia el aporte brindado por los pobladores de las distintas comunidades visitadas, ya que eran ellos quienes reportaban la presencia de material arqueológico en superficie, además de posibles sitios con montículos dentro y fuera del municipio, lo que refleja mucho material en la zona.

Todos los sitios documentados durante este inventario y los diferentes reportes, ya sean de evidencias o de posibles lugares con materiales arqueológicos, prueban la gran riqueza arqueológica existente en la región. Esto corrobora igualmente que este territorio estuvo poblado antiguamente, por lo que es preciso que estos sitios $\mathrm{y}$ materiales sean registrados y documentados como patrimonio arqueológico.

Esta investigación no es definitiva dado que es muy probable que existan otros sitios en el municipio que aún no han sido documentados; y más todavía, porque este proyecto contempló prospectar comunidades ya seleccionadas con anterioridad y no todas las comunidades del municipio en donde puede encontrarse más material arqueológico. En este sentido es importante conocer, por ejemplo, el análisis detallado del material cerámico y lítico. También es necesario un estudio sistemático de las estructuras monticulares existentes, por lo cual seria conveniente plantear una excavación arqueológica que permita reforzar aún más los estudios arqueológicos en la zona.

En síntesis cabe destacar que esta investigación corresponde únicamente a la documentación y registró de sitios arqueológicos, así como también a la descripción, la clasificación y análisis de las muestras. También, con el material cerámico se logró realizar algunas asociaciones en cuanto a similitudes y patrones en técnicas de decoración con sitios documentados en el área de Centroamérica y con sitios estudiados en el Caribe Sur de nuestro país.

\section{RECOMENDACIONES}

Como resultado de esta investigación, se plantean las siguientes recomendaciones:

- De manera general, las recomendaciones van dirigidas principalmente al periodo en que deben 
hacerse las prospecciones arqueológicas, dado que la lluvia, por ejemplo, es un factor que afecta el trabajo de prospección, puesto que en el invierno crecen la maleza y los arbustos, lo cual dificulta la observación en los sitios donde se encuentran las evidencias.

- Con la cerámica recolectada de los diferentes sitios registrados durante esta prospección se recomienda hacer un estudio dirigido específicamente a la clasificación tipológica de estas piezas, con el fin de establecer estimaciones cronológicas de los sitios.

- Se recomienda continuar y ampliar el radio de acción de las prospecciones arqueológicas, con el objetivo de cubrir todo el territorio que comprende el municipio de Rosita.

- También es importante destacar que es necesario hacer prospecciones arqueológicas en la zona de Kukalaya, dado que durante esta prospección fueron entrevistadas personas que refieren que en esas zonas la presencia de evidencias arqueológicas es mucha.

- Finalmente recomendamos implementar un plan de sensibilización dirigido a destacar la importancia que tiene el patrimonio arqueológico para el Municipio.

\section{AGRADECIMIENTOS}

Para que esta tarea fuese exitosa se contó con el respaldo y colaboración de los pobladores del municipio de Rosita, de la Alcaldía Municipal, docentes universitarios y compañeros que se desempeñaron en tareas como informantes y baqueanos: Marco Adán Silva, Julio Torrez Ruiz, Felícito Paz Cantillano, María Antonia Ríos, Ronald Reyes, Michael Rostran, Noel Palacio, José Mariano, Román Amador, Cosme Lopez, Jorge Jiménez y Arcenio García.

\section{BIBLIOGRAFIA}

- Aguerre, A. M. \& Lanata, J. L. (comps.). (2005). Explorando algunos temas de arqueología. Barcelona: Gedisa.

- Arrechavala Romero, J. (2007). Historia de la Costa Caribe de Nicaragua.Managua: Acento.

- Enciclopedia de Nicaragua, vol.1.(2002). Barcelona: OCEANO.

- Esgueva Gómez, A.(1996). La Mesoamérica nicaragüense: Documentos y comentarios. Managua: UCA.

- Ferrero Acosta, L. (2000). Costa Rica precolombina.San José: Editorial Costa Rica.

- González Hodgson, K. \& Taylor Rigby, E. (2009). Arqueología en la RAAN: Una aproximación al pasado. Monografía de licenciatura no publicada, UNAN, Managua.

- Latino Muñoz, B. C. (2005). Alfarería en la costa Caribe de Nicaragua, Kukra Hill: Caracterización morfométrica, tecnológica y de uso de artefactos cerámicos procedentes del sitio Karoline, Conchero KH-4. Unidades estratigráficas 1006 y 2006 (250-350 cal. DNE). Monografía de licenciatura no publicada, Managua.

- Renfrew, C. \& Bahn, P. (1993). Arqueología: Teoría, métodos y práctica. Madrid: Akal.

- Salazar, J. (2008). Aportes de Gordon R. Willey a la comprensión histórica de la arqueología americana. Comechingonia Virtual, Revista electrónica de arqueología. (4), 245-254.

- Smutko, G. (1985). La mosquitia: Historia y cultura de la Costa Atlántica. Managua: La Ocarina.

- Wheeler, M. (1961). Arqueología de campo. México: Fondo de Cultura Económica.

\section{WEBGRAFIA}

- http://www.inifom.gob.ni/municipios/documentos

- www.bio-nica.info/biblioteca/AnonimoGeologiaSintesisGeologia 